\title{
PerformanCE OF Wireless OFDM SYSTEM WITH LS-INTERPOLATION-BASED CHANNEL Estimation in Multi-PATH FAding Channel
}

\author{
A.Z.M. Touhidul Islam and Indraneel Misra \\ Department of Information and Communication Engineering \\ University of Rajshahi, Rajshai-6205, Bangladesh \\ touhid_ict_ru@yahoo.com, misraindraneeleyahoo.com
}

\begin{abstract}
In this paper we investigate the bit error rate (BER) performance of orthogonal frequency division multiplexing (OFDM) wireless communication system with the implementation of LS-Interpolation-based comb-type pilot symbol-assisted channel estimation algorithm over frequency selective multi-path Rayleigh fading channel. The Least square (LS) method is used for the estimation of channel at pilot frequencies while different interpolation techniques such as low-pass interpolation, cubic interpolation, spline cubic interpolation, linear interpolation and FFT interpolation are employed to interpolate the channel at data frequencies. In signal mapping, the OFDM system incorporates M-ary phase-shift keying (M-PSK) and Mary quadrature amplitude modulation (M-QAM) digital modulation schemes. Matlab simulations are carried out to analyze the performance of the developed OFDM system with the employment of comb-pilot based channel estimation algorithms for various digital modulations in Rayleigh fading environment. The impact of Doppler frequency and number of channel taps on the BER performance is also investigated.
\end{abstract}

\section{KEYWORDS}

Channel estimation, Orthogonal frequency division multiplexing (OFDM), LS estimation, Interpolation, Comb-Pilot.

\section{INTRODUCTION}

As a multicarrier transmission system Orthogonal frequency division multiplexing (OFDM) has been widely applied in wireless communication systems due to its numerous advantages like robustness against multipath fading and delay, high data rate transmission capability, high spectral efficiency, allows adaptive modulations and coding of subcarriers, and it provides an efficient way of eliminating inter-symbol interference (ISI) for transmission over frequency selective multi-path fading channels [1,2]. However, as the channel transfer function of radio channel in OFDM systems looks unequal in both the time and frequency domains, a dynamic estimation of the channel is necessary. Furthermore, the estimators should have both low complexity and high accuracy [3]. The two basic 1D channel estimations adopted in OFDM systems are block-type (pilots insert in the frequency direction) and comb-type (pilots insert in the time direction) pilot-based channel estimations. In block-type pilot arrangement, the pilot signal is assigned to particular OFDM block and sent periodically in time domain. Because the training block contains all pilots, channel interpolation in frequency domain is not required in 
block-type pilot channel estimation and the estimation of the channel can be based on Least Square (LS) or Minimum Mean-Square Error (MMSE).

On the other hand, in comb-type pilot arrangement, the pilot signals are uniformly distributed within each OFDM block. The comb-type pilot-based channel estimation (can be introduced to satisfy the need for equalization [4]) consists of algorithms to estimate the channel at pilot frequencies and to interpolate the channel at data frequencies. The LS, MMSE or Least MeanSquare (LMS) method can be used to estimate the channel at pilot frequencies, while different interpolation schemes are introduced for the channel estimation at data frequencies $[5,6]$.

In this paper, our aim is to evaluate the bit error rate (BER) performance of OFDM wireless communication system with the implementation of comb-type pilot assisted channel estimation algorithms by incorporating M-PSK and M-QAM digital modulation schemes over Rayleigh frequency selective multi-path fading channel. The LS estimator is employed because of its lowest complexity. In addition, the performance of different interpolation techniques such as lowpass, cubic, spline cubic, linear and FFT are also examined.

\section{RELATED WORKS}

In earlier studies [7]-[9] different pilot-based channel estimation in OFDM wireless communication systems in the time and frequency domains was investigated. Performance of different Interpolating schemes were examined in $[4,10]$. X. Cai and G.B. Giannakis [11] studied the performance of OFDM system with M-PSK digital modulation over Raleigh frequency selective multi-path fading channel in presence of channel estimation errors and performed optimization of the number of pilot symbols, the placement of pilot symbols and the power allocation between pilot and information symbols in order to minimize the performance loss. Reference [4] presented a review of block type and comb-type pilot based channel estimation and showed that comb-type pilot based channel estimation with low pass interpolation performs the best among all channel estimation algorithms.

The performance and complexity of two basic channel estimation algorithms such as Least Square error (LSE) and Linear Minimum Mean Square error (LMMSE) are evaluated based on channel impulse response (CIR) samples and channel taps [12] and showed that LSE can be made more efficient than LMMSE by increasing CIR samples. H.M. Mohmoud et al. [13] explored comb-type pilot aided channel estimation and proposed Kalman and LS estimators to estimate the channel frequency response (CFR) at the pilot location. The authors in reference [14] shown that the number of pilot symbols for a desired BER and Doppler frequency is highly dependent on the pilot pattern used.

\section{SYSTEM DESCRIPTION}

Figure 1 shows a typical block diagram of wireless OFDM system with pilot signal assisted channel estimation. The information data in binary form are first grouped and mapped into multiamplitude multi-phase signals according to the type of modulation (M-PSK and M-QAM) used in the signal modulator. After inserting pilots uniformly between the information data sequence, IFFT block is used to transform and multiplex the complex data sequence into time domain signal. Following the IFFT block, a guard interval (larger than the expected delay spread), is inserted in order to prevent possible inter-symbol interference (ISI) in OFDM systems. The transmitted signal is then sent to a frequency selective multi-path time varying Rayleigh fading channel. 
At the receiver, the guard interval is removed first and the received samples are then sent to an FFT block for de-multiplexing the multi-carrier signals. Following FFT block, the pilot signals are extracted from the demultiplexed samples. The transmitted data samples can then be recovered from the knowledge of the channel responses by simply dividing the received signal by the channel response $[15,16]$. After signal demodulation at the demodulator, the source binary data could be reconstructed at the receiver output.

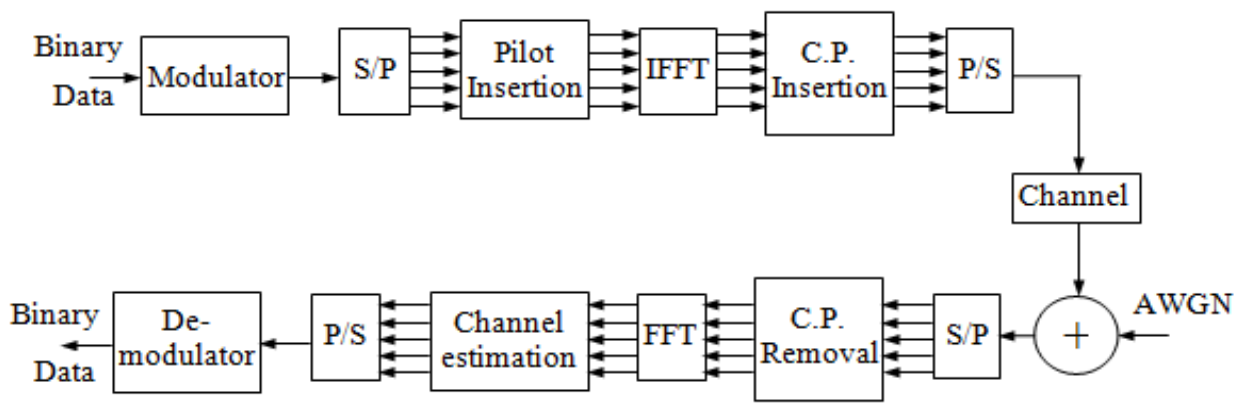

Fig. 1 Block diagram of OFDM system with pilot-based channel estimation.

\section{COMb-TYPE PILOT SYMbOL-ASSISTED CHANNEL ESTIMATION}

For channel estimation some kinds of pilot symbol is necessary as a point of reference which allow the receiver to extract channel attenuations and phase rotation estimates for each received symbol and facilitates their compensation. In Pilot symbol assisted modulation (PSAM), channel estimates are achieved by multiplexing pilot symbols into data sequence [17]. Here we consider comb-type pilot arrangement (can provide better resistance to fast-fading channels) in which the pilot signals are uniformly distributed within each OFDM block as shown in Fig. 2. In comb-type pilot-aided channel estimation algorithm shown in Fig.3, the pilot signals are first extracted from the received signal. The channel transfer function is then estimated from the received pilot signals and the known pilot signals $[1,18]$. Because only few sub-carriers in comb-type pilot arrangement contain pilot signals, the channel responses of non-pilot subcarriers (carry data) can be estimated by interpolating the neighboring pilot channel responses.

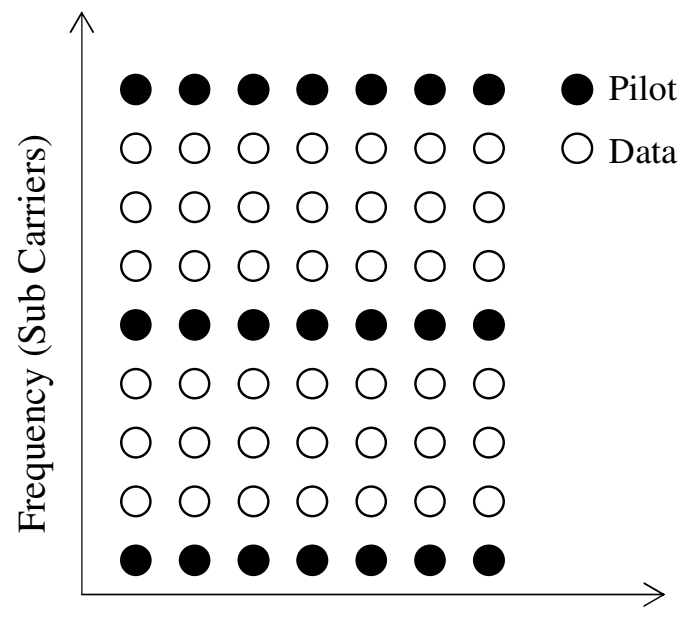

Time (OFDM Symbols)

Fig. 2 Comb-type pilot-symbol arrangement. 
Although comb-type pilot based channel estimation can be based on least square (LS), minimum mean square error (MMSE) or least mean square (LMS) [4], in this paper, we considered only simple, low complexity LS channel estimation algorithm for the estimation of channel at pilot subcarriers. Pilot signal estimation and channel interpolation algorithms are discussed in the following subsections.

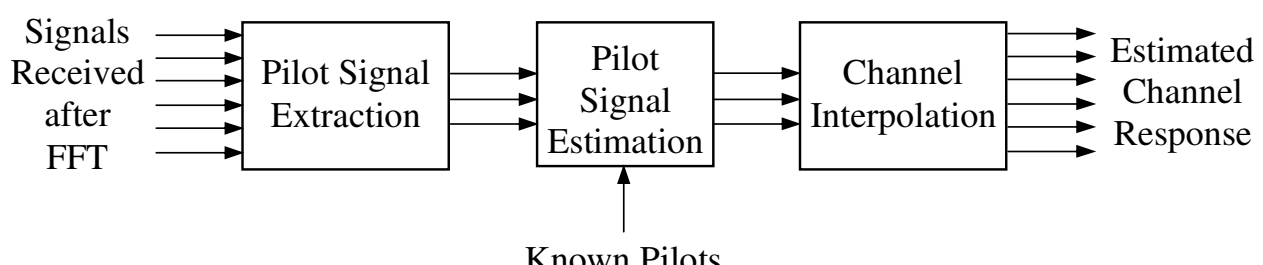

Fig. 3 channel estimation algorithm based on Comb-type pilot arrangement.

\subsection{Least square channel estimation at pilot subcarriers}

Suppose $M_{p i}$ pilot signals $X_{p i}(n), n=0,1, \cdots \cdots, M_{p i}-1$ are uniformly inserted into $X(k)$ data signals. The OFDM signal modulated on the $k$-th subcarrier can be expressed as

$$
\begin{aligned}
X(k) & =X\left(n L_{s}+i\right) \\
& =\left\{\begin{array}{c}
X_{p i}(n), i=0 \\
\text { Source data }, i=1,2, \cdots \cdots, L_{s}-1
\end{array}\right.
\end{aligned}
$$

when the total $M$ subcarriers are divided into $M_{p i}$ groups, each with $L_{s}=M / M_{p i}$ adjacent subcarriers. The estimate of pilot signals based on least squares (LS) criterion is given by [6]

$$
\hat{H}_{p i}=X_{p i}^{-1} Y_{p i}
$$

where

$$
H_{p i}=\left[H_{p i}(0) H_{p i}(1) \cdots \cdots \cdots \cdot H_{p i}\left(M_{p i}-1\right)\right]^{T}
$$

the channel frequency response at pilot sub-carriers,

$$
Y_{p i}=\left[Y_{p i}(0) Y_{p i}(1) \cdots \cdots \cdots Y_{p i}\left(M_{p i}-1\right)\right]^{T}
$$

the received pilot signals vector which can also be expressed as

$$
\begin{gathered}
Y_{p i}=X_{p i} \cdot H_{p i}+I_{p i}+W_{p i} \\
\text { where } X_{p i}=\left[\begin{array}{ccc}
X_{p i}(0) & & 0 \\
& \ddots & \\
0 & & X_{p i}\left(M_{p i}-1\right)
\end{array}\right], I_{p} \text { and } W_{p} \text { are the inter-carrier }
\end{gathered}
$$

interference (ICI) vector and the Gaussian noise vector in pilot subcarriers, respectively.

\subsection{Channel Interpolation techniques at data subcarriers}

We need an efficient interpolation technique in order to estimate channel at data subcarriers by using the channel information at pilot subcarriers. Here we considered the following interpolation techniques: linear interpolation, spline cubic interpolation, cubic interpolation, FFT interpolation and low-pass filter interpolation. Two successive known pilot subcarriers are used in linear interpolation to determine the channel response for data subcarriers that are located in between the pilots [19] and the algorithm can be realized using digital filtering such as the Farrow- 
structure [20]. The intermediate estimates are evaluated by the linear sum of the known components on either side. Spline cubic interpolation is based on drawing smooth curves through a number of points which produces smooth and continuous polynomial fitted to given data points [21]. The cubic interpolation uses four known points to obtain a third degree polynomial [22]. In case the range of interpolation becomes larger than the range covered by the first four reference points, it is required to obtain a second polynomial using the next four points. The basic principle of FFT interpolation is to apply the FFT in the data to be interpolated, the null samples are added in the frequency domain and the oversampled vector is applied to the IFFT. In low-pass interpolation, zeros are inserted into the original sequence [4], low-pass finite impulse response (FIR) filter is applied to allow the original data to pass through unchanged and interpolates between such that the mean-square error between the interpolated points and their ideal values is minimized.

\section{Performance Results and Discussion}

The computer simulation has been performed using Matlab 7.5 programming language and the parameters used in the simulation are listed in Table 1. The simulation results are presented in graphical form Figures 4 to 8 . Number of channel taps and Doppler frequency for the results in Figs. 4-6 are considered as 16 and $100 \mathrm{~Hz}$, respectively.

Table 1. Simulation Parameters

\begin{tabular}{|c|c|}
\hline Parameter & Specification \\
\hline Number of Sub-carrier & 256 \\
\hline Number of Pilot & 32 \\
\hline Pilot Ratio & $1 / 8$ \\
\hline Pilot-to-Data Power Ratio & 2 \\
\hline IFFT, FFT Size & 256 \\
\hline Guard Interval & 64 \\
\hline Modulation Type & M-PSK, M-QAM \\
\hline Channel Model & Rayleigh Fading \\
\hline Number of Channel Taps & $2-64$ \\
\hline Doppler Frequency & $20-280 \mathrm{~Hz}$ \\
\hline
\end{tabular}

Figure 4 shows the BER performance of the BPSK-modulated OFDM system in Rayleigh fading channel where channel estimation at data locations was obtained by using FFT interpolation technique. It is seen that with the use of no LS estimator error rate at the receiver is very high. The BER performance of the OFDM system has greatly improved with the use of Comb-type pilot-aided LS channel estimation algorithm which results from the decrease of the amplitude and phase distortion caused by multipath distortion.

The BER performance of the OFDM system under M-PSK (2-PSK, 4-PSK, 8-PSK and 16-PSK) and M-QAM (2-QAM, 4-QAM, 8-QAM and 16-QAM) digital modulations over Rayleigh fading channel is shown in Fig. 5. The FFT interpolation technique is used to estimate CFR at data frequencies. Simulation results show that 2-PSK (or BPSK) modulation has achieved better error rate performance than 4-PSK, 8-PSK and 16-PSK. Moreover, 2-QAM has given better performance than 4, 8 and 16-QAM modulations. The OFDM system outperforms at BPSK modulation and shows worst performance at 16-QAM. For a typical SNR value of $10 \mathrm{~dB}$, the bit error rate for BPSK and 16-QAM are 0.0349 and 0.8389 respectively which implies that with the use of BPSK modulation the system performance is improved by $13.81 \mathrm{~dB}$. 


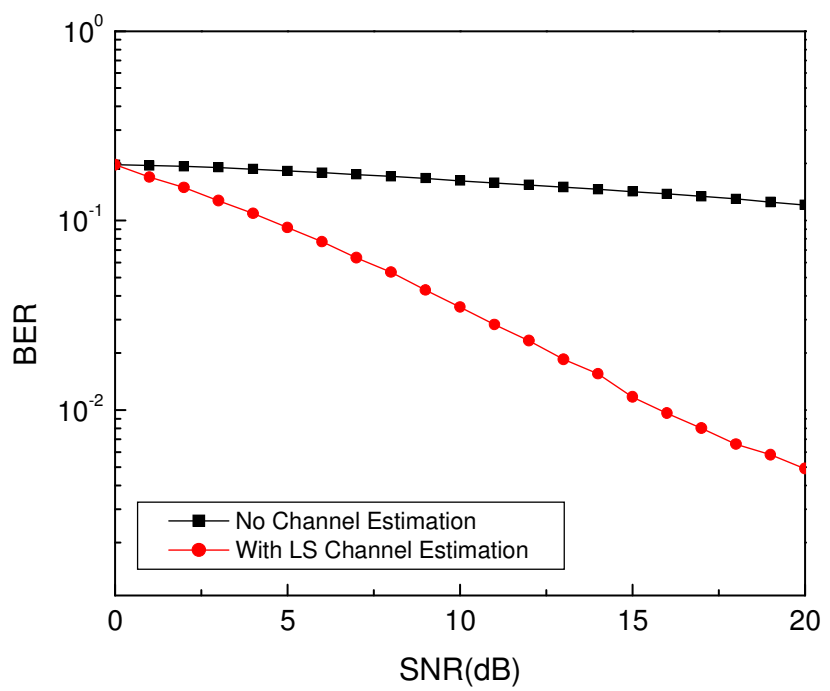

Fig. 4 Effect of comb-pilot based LS channel estimation on the BER performance of BPSK-modulated OFDM system .

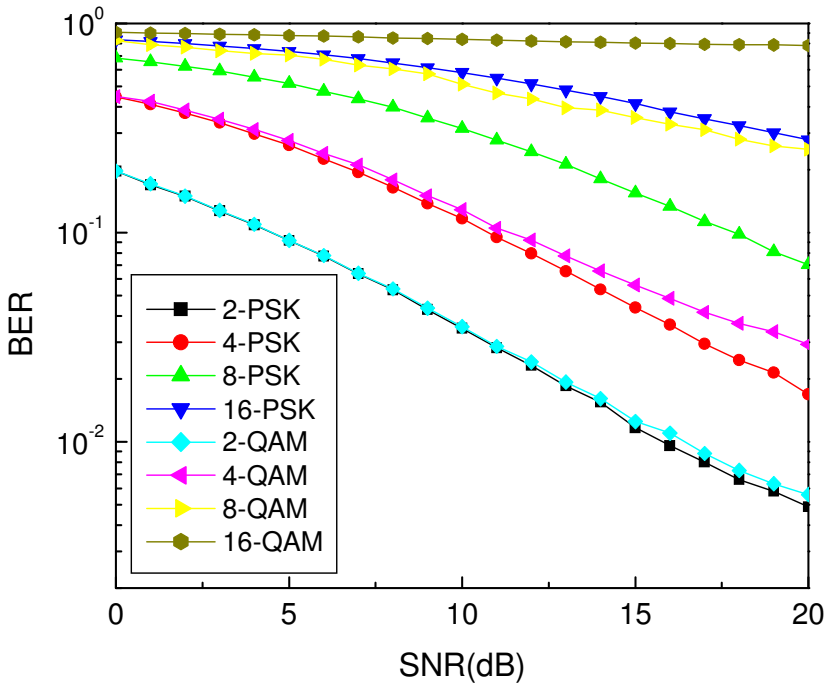

Fig.5 BER performance of M-PSK and M-QAM-modulated OFDM system with Comb-pilot based channel estimation.

The impact of different channel interpolation algorithms on the performance of the OFDM system shown in Fig. 6. The simulation is performed under BPSK modulation over Rayleigh fading channel. It is evident that Comb-type LS channel estimation with low-pass interpolation achieves the best error rate performance than other interpolation algorithms used while the linear interpolations shows worst performance in CFR estimation. It may results from the fact that low- 
pass interpolation does minimization of the mean-square error between the interpolated points and their ideal values $[4,10,15]$.

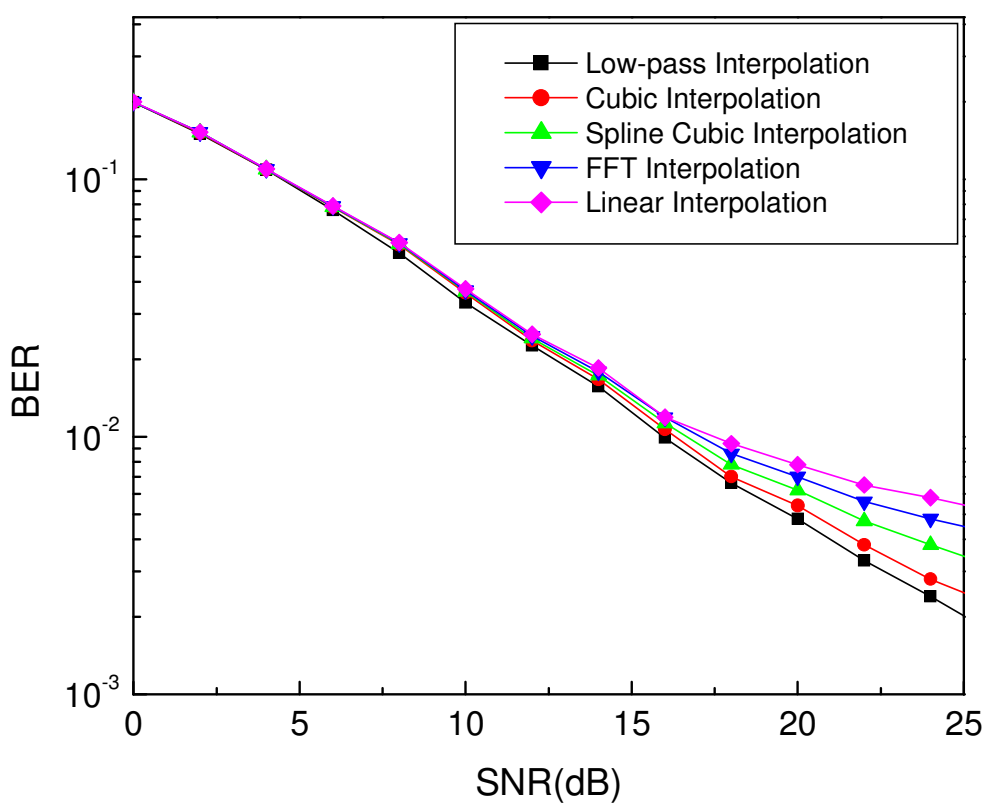

Fig.6 Impact of different interpolation techniques on the BER performance of BPSK-modulated OFDM system.

The effect of Doppler frequency shift on the BER performance of the BPSK-modulated OFDM system over Rayleigh fading channel is shown in Fig. 7. Channel estimation at data locations was obtained using FFT interpolation. The pilot-to-data power ratio, number of channel taps and the SNR value are considered as 2, 16 and $15 \mathrm{~dB}$, respectively. It is observed that at a fixed SNR the bit error rate remains nearly constant and slightly increase with the variations in Doppler frequency. It may results from the presence of inter-channel interference [23] and the use of smaller length of guard interval than the number of sub-carriers in the simulation [13].

Fig. 8 shows the effect of additional channel taps on BER performance of BPSKmodulated OFDM system under Rayleigh fading channel. Pilot-to-data power ratio, Doppler frequency, channel interpolation algorithms and SNR value are considered as 2, $100 \mathrm{~Hz}$, FFT and $10 \mathrm{~dB}$, respectively. It is evident that the channel uncertainty increases with increasing number of unknown channel taps in OFDM frequency selective multipath fading channel [24]. 


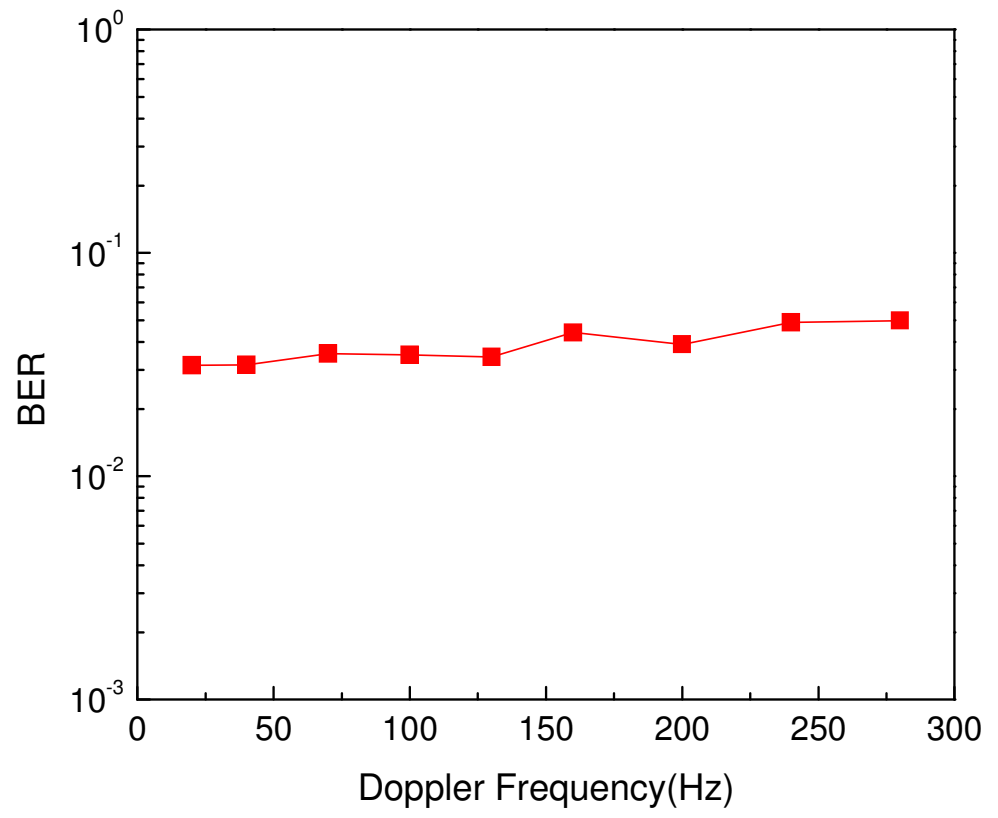

Fig.7 Dependence of BER Performance of BPSK-modulated OFDM system on Doppler frequency shift at SNR $15 \mathrm{~dB}$.

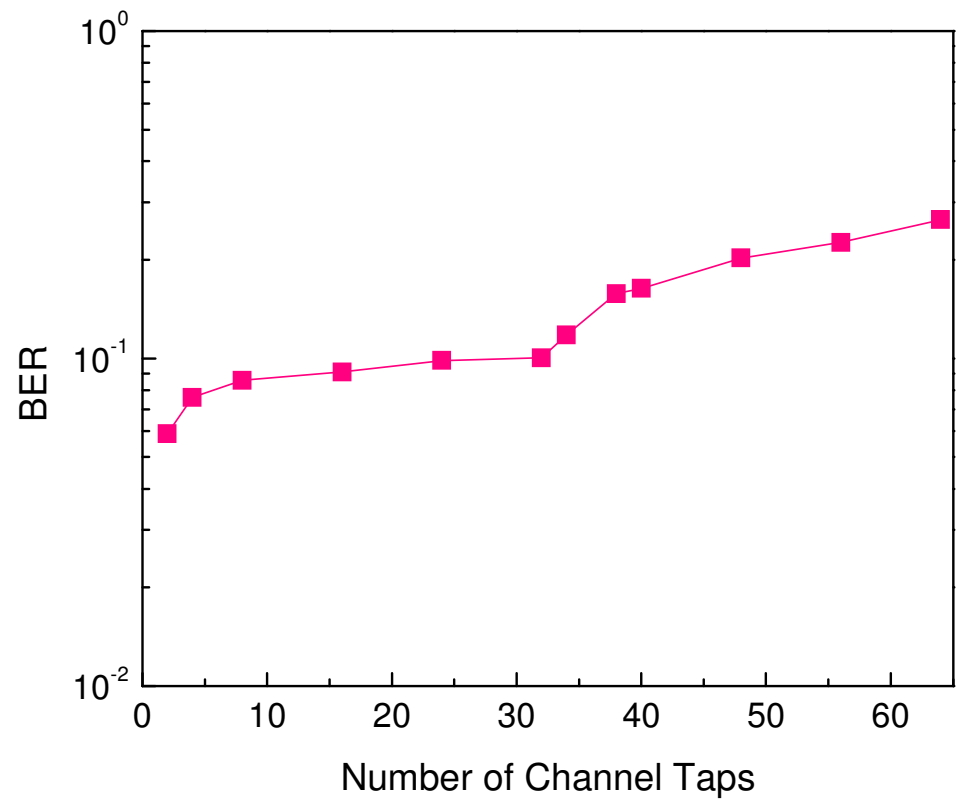

Fig. 8 Influence of Channel Taps on the BER performance of BPSK-modulated OFDM system at SNR 10dB. 
International Journal on Computational Sciences \& Applications (IJCSA) Vo2, No.5, October 2012

\section{Conclusions}

In this paper we investigated the BER performance of M-PSK and M-QAM-modulated OFDM wireless communication systems with the implementation of LS-Interpolation-based comb-type pilot symbol-assisted channel estimation algorithm over frequency selective multi-path Rayleigh fading channel. In channel estimation, the OFDM system employed Least square estimator for the estimation of channel at pilot frequencies while different interpolation techniques are used to interpolate the channel at data frequencies. Simulation results show that the proposed OFDM system with LS channel estimator achieves good error rate performance under the BPSK and 2QAM modulation schemes over Rayleigh fading channel. Low-pass interpolation performs better in channel frequency response estimation than other studied interpolation algorithms and the BER performance of OFDM system with comb pilot-assisted channel estimation is less affected by Doppler frequency.

\section{REFERENCES}

[1] O. Edfors, M. Sandell, J.-J. Van de Beek, D. Landström and F. Sjöberg, "An Introduction to Orthogonal Frequency Division Multiplexing, Luleå Sweden: Luleå Tekniska Universitet, pp. 1-58, 1996.

[2] Z. Wang and G.B. Giannakis, "Wireless Multicarrier Communications: where Fourier meets Shannons,” IEEE Signal Processing Mag., Vol. 47, No. 3, pp. 29-48, May 2000.

[3] A.R.S. Bahai and B.R. Saltzberg, "Multicarrier Digital Communications: Theory and Applications of OFDM: Kluwer Academic, Plenum, 1999.

[4] S. Coleri, M. Ergen, A. Puri and A. Bahai, "Channel Estimation Techniques Based on Pilot Arrangement in OFDM Systems," IEEE Transactions on Broadcasting, Vol. 48, No. 3, Sep. 2002.

[5] J.H. Kotecha and A.M. Sayeed, Transmit signal design for optimal estimation of correlated MIMO channels, "IEEE Transactions on Signal Processing, Vol. 52, pp. 546-557, Feb. 2004.

[6] C. Chuah, D.N.C. Tse and J.M. Kahn, et al., "Capacity scaling in MIMO wireless systems under correlated fading," IEEE Trans. on Information Theory, Vol. 48. No. 3, pp. 637-650, 2002.

[7] P. Höecher, S. Kaiser and P. Robertson, "Two-dimensional pilot-symbol-aided channel estimation by wiener filtering," In Proc. Int. Conf. Acoust., Speech and Signal Processing, Munich Germany, pp. 1845-1848, Apr. 1997.

[8] Y.G. Li, "Pilot -symbol-aided channel estimation for OFDM in wireless systems," IEEE Trans. Veh. Technol., Vol. 49, pp. 1207-1215, July 2000.

[9] Y.G. Li, L.J. Cimini and N.R. Sollenberger, "Robust channel estimation for OFDM system with rapid diverse fading channels," IEEE Trans. Commun., Vol. 46, pp. 902-914, July 1998.

[10] M. Hsieh and C. Wei, "Channel estimation for OFDM systems based on comb-type pilot arrangement in frequency selective channels," IEEE Trans. Consumer Electron., Vol. 44, pp. 217-225, Feb. 1998.

[11] X. Cai and G.B. Giannakis, "Error probability minimizing pilots for OFDM with M-PSK modulation over Rayleigh fading channels," IEEE Trans. Vehicular Techn., Vol. 53, No. 1, pp. 146-155, Jan. 2004.

[12] S. Saleem and Q.-Ul-Islam, "Performance and complexity comparison of channel estimation algorithms for OFDM system," International Journal of Electrical and Computer Sciences, Vol. 11, No. 2, pp. 6-12, Apr. 2011.

[13] H.M. Mahmoud , A.S. Mousa and R. Saleem, "Channel estimation based on comb-type pilots arrangement for OFDM system over time varying channel,” Journal of Networks, Vol. 5, No. 7, July 2010.

[14] F. Tufvesson and T. Maseng, "Pilot assisted channel estimation for OFDM in mobile cellular systems," Proceedings of IEEE Vehicular Tech. Conference, Phoenix USA, pp. 1639-1643, May 1997.

[15] R. Steele, Mobile Radio Communications, England: Pentech Press Limited, 1992.

[16] Y. Zhao and A. Huang, "A novel channel estimation method for OFDM mobile communication systems based on pilot signals and transform domain processing," in Proc. IEEE $47^{\text {th }}$ Vehicular Technology Conference, Phoenix, USA, pp. 2089-2093, May 1997. 
International Journal on Computational Sciences \& Applications (IJCSA) Vo2, No.5, October 2012

[17] J. Torrance and L. Hanzo, "Comparative study of pilot symbol assisted modem systems," Proceedings of IEEE Conf. Radio Receivers and Associated Systems, Bath UK, pp. 36-41, Sept. 1995.

[18] J.-J. van de Beek, O. Edfors, M. Sandell, S.K. Wilson and P.O. Börjesson, "On channel estimation in OFDM systems," in Proc. IEEE $45^{\text {th }}$ Vehicular Technology Conference, Chicago IL, USA, pp. 815 819, Jul. 1995.

[19] J. Rinne and M. Renfors, "Pilot spacing in orthogonal frequency division multiplexing systems on practical channels," IEEE Transactions on Customer Electronics, Vol. 42, no. 4, Nov. 1996.

[20] C.W. Farrow, "A continuously variable digital delay element," in Proc. IEEE Int. Symp. Circuits and Syst., Espoo, Finland, pp. 2641-2645, June 6-9, 1988.

[21] Y. Shen and Ed Martinez, "Channel estimation in OFDM systems," Free Scale Semiconductor, AN3059 Inc., 2006.

[22] C.A. Rocha, L.L. Mendes and A.C. da Silveira, "Performance analysis of channel estimation schemes for OFDM systems," Proc. of the International Workshop on Telecommunications, pp. 32-36, IWT/07.

[23] R. Vann Nee and R. Prasad, “OFDM for Wireless Multimedia Communications,” Artech House Publishers, Massachusetts, 2000.

[24] E. Zeydan and U. Tureli, "Differential Space-Frequency Group Codes for MIMO-OFDM," 41st Annual Conference on Information Sciences and Systems, pp. 542 - 547, 14-16 March 2007. 